\title{
Association of Body Composition with Long-Term Survival in Non-metastatic Rectal Cancer Patients
}

\section{Jin Soo Han, MD ${ }^{1}$ \\ Hyoseon Ryu, MD'1 \\ In Ja Park, MD, PhD' \\ Kyung Won Kim, PhD2 \\ Yongbin Shin, MS² \\ Sun Ok Kim ${ }^{3}$ \\ Seok-Byung Lim, $\mathrm{PhD}^{1}$ \\ Chan Wook Kim, PhD' \\ Yong Sik Yoon, $\mathrm{PhD}^{1}$ \\ Jong Lyul Lee, PhD' \\ Chang Sik Yu, PhD \\ Jin Cheon Kim, PhD'}

\section{${ }^{1}$ Department of Colon and Rectal Surgery ${ }^{2}$ Department of Radiology and Research Institute of Radiology, ${ }^{3}$ Department of Clinical Epidemiology and Biostatistics, Asan Medical Center, University of Ulsan College of Medicine, Seoul, Korea}

Correspondence: In Ja Park, MD, PhD Department of Colon and Rectal Surgery, Asan Medical Center, University of College of Medicine, 88 Olympic-ro 43-gil, Songpa-gu,

Seoul 05505, Korea

Tel: 82-2-3010-3937

Fax: 82-2-3010-6701

E-mail: ipark@amc.seoul.kr

Received May 7, 2019

Accepted November 20, 2019

Published Online December 3, 2019

\begin{abstract}
Purpose
We evaluated the association of body composition with long-term oncologic outcomes in non-metastatic rectal cancer patients.
\end{abstract}

\begin{abstract}
Materials and Methods
We included 1,384 patients with stage(y)0-III rectal cancer treated at Asan Medical Center between January 2005 and December 2012. Body composition at diagnosis was measured using abdomino-pelvic computed tomography (CT). Sarcopenia, visceral obesity (VO), and sarcopenic obesity (SO) were defined using CT measured parameters such as skeletal muscle index (total abdominal muscle area, TAMA), visceral fat area (VFA), and VFA/TAMA. Inflammatory status was defined as a neutrophil-lymphocyte ratio of $\geq 3$. Obesity was categorized by body mass index $\left(\geq 25 \mathrm{~kg} / \mathrm{m}^{2}\right)$.
\end{abstract}

\section{Results}

Among the 1,384 patients, 944 (68.2\%) had sarcopenia and 307 (22.2\%) had SO. The 5 -year overall survival (OS) rate was significantly lower in sarcopenic patients (no sarcopenia vs. sarcopenia; $84 \%$ vs. $78 \%, p=0.003$ ) but the 5 -year recurrence-free survival (RFS) rate was not different (77.3\% vs. $77.9 \% \mathrm{p}=0.957)$. Patients with SO showed lower 5 -year OS (79.1\% vs. $75.5 \% p=0.02$ ) but no difference in 5-year RFS ( $p=0.957)$. Sarcopenia, SO, VO, and obesity were not associated with RFS. However, obesity, SO, age, sex, inflammatory status, and tumor stage were confirmed as independent factors associated with OS on multivariate analysis. In subgroup analysis, association of SO with OS was more prominent in patients with (y)p stage 0-2 and no inflammatory status.

\section{Conclusion}

The presence of $\mathrm{SO}$ and a low body mass index at diagnosis are negatively associated with OS in non-metastatic rectal cancer patients.

\section{Introduction}

Identification of potentially modifiable factors associated with oncologic outcomes and treatment response in patients with cancer is clinically important. In this context, body composition parameters associated with fat and muscle distribution has gained increased attention [1,2]. Besides this, systemic inflammation is a speculated risk factor affecting the survival of cancer patients [3,4].

\section{Key words}

Rectal neoplasms, Sarcopenia, Obesity, Overall survival, Recurrence-free survival 
inflammation affect overall survival (OS) and recurrence-free survival (RFS) in CRC patients [10]. Studies reporting sarcopenic patients with colorectal cancer have higher levels of systemic inflammatory markers and increased systemic inflammation affects survival in these cases has furthered the interest in these parameters [11-13]. SO, in which excessive body fat is present alongside low muscle mass and quality, has also been shown to be associated with decreased survival in colorectal cancer [14-16]. However, studies evaluating the influence of various body composition parameters on oncologic outcomes in colorectal cancer are limited.

Since sarcopenia, SO, and inflammatory markers can be readily measured by routine abdominopelvic computed tomography $(\mathrm{CT})$ and laboratory blood tests prior to surgery and are correctable by exercise and medication, their correlation with negative oncological outcomes would be clinically significant. In the present study, we aimed to examine whether body composition parameters and systemic inflammation affect oncological outcomes in rectal cancer patients.

\section{Materials and Methods}

\section{Study population}

We enrolled stage (y)0-III rectal cancer patients who received surgical treatment at Asan Medical Center from January 2005 to December 2012. All of the included study patients underwent abdominopelvic CT and blood testing during their first diagnosis. For patients who had received preoperative chemoradiotherapy (PCRT), test records at the time of diagnosis, i.e., prior to this treatment, were analyzed. Data on the body mass index (BMI), age, sex, tumor stage, PCRT, recurrence, survival duration, and laboratory findings for the study patients were collected via a review of their medical records.

\section{Body composition parameters}

All CT images were retrieved from the Picture Archiving and Communication System at our institution. The presence of sarcopenia was evaluated on abdominal CT using Asan-J software, based on ImageJ (NIH, Bethesda, MD). Two consecutive axial CT images at the level of the inferior endplate of the L3 lumbar vertebra were processed and averaged for each patient. Using Asan-J, the total abdominal muscle area (TAMA, $\mathrm{cm}^{2}$ ), including all muscles on selected axial images, i.e., psoas, paraspinals, transversus abdominis, rectus abdominis, quadratus lumborum, and internal and external obliques, were demarcated using predetermined Hounsfield unit

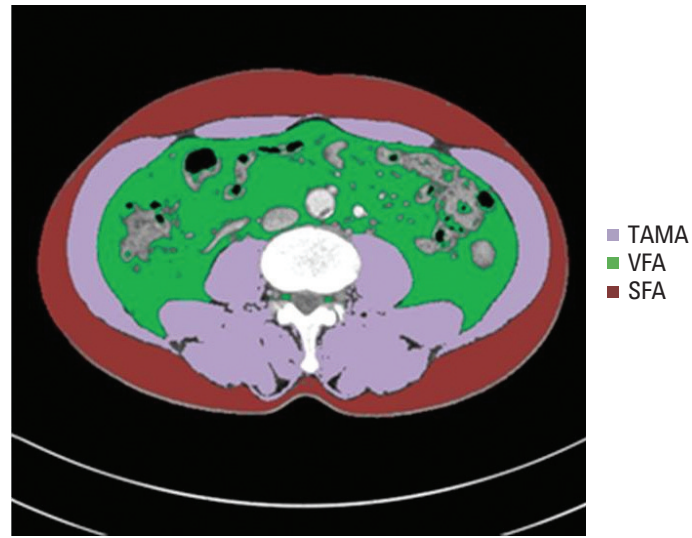

Fig. 1. Body morphometric evaluations of abdominal fat and muscle areas. At the level of the inferior endplate of the L3 vertebra, an axial computed tomography image was segmented into the total abdominal muscle area (TAMA), visceral fat area (VFA), and subcutaneous fat area (SFA).

thresholds on $\mathrm{CT}$ or the signal intensity on precontrast images [17]. The visceral fat area $\left(\mathrm{VFA}, \mathrm{cm}^{2}\right)$ and subcutaneous fat area $\left(\mathrm{SFA}, \mathrm{cm}^{2}\right)$ were demarcated using the adipose tissue thresholds on CT (Fig. 1). Visceral obesity (VO) was defined as a VFA $\geq 100 \mathrm{~cm}^{2}$. The skeletal muscle index (SMI) was calculated as TAMA/height ${ }^{2}$ and sarcopenia was defined by an SMI of $\leq 52.4 \mathrm{~cm}^{2} / \mathrm{m}^{2}$ for men and $\leq 38.5 \mathrm{~cm}^{2} /$ $\mathrm{m}^{2}$ for women [8]. A VFA/TAMA ratio of above 3.2 was defined as SO [18]. The BMI was calculated as weight $(\mathrm{kg}) /$ height ${ }^{2}\left(\mathrm{~m}^{2}\right)$. A BMI above 25 was considered obesity.

\section{Marker of systemic inflammation}

We used neutrophil-lymphocyte ratio (NLR) as an indicator of systemic inflammation. A NLR of 3 or greater was considered to indicate an inflammatory status and values below 3 , of no inflammatory status $[10,19]$.

\section{Statistical analysis}

All continuous variables were shown as mean values with a standard deviation. Survival duration was determined from the time of first diagnosis to the period of death from any cause or the last follow-up date. In the RFS analysis, the time of disease recurrence or death from any cause was considered. The associations between sarcopenia and $\mathrm{SO}$ and the 5 -year OS and RFS outcomes in our rectal cancer cohort were analyzed using Kaplan-Meier curves. Cox regression analysis was used to evaluate the factors associated with patient survival duration, and calculate the hazard ratios (HRs) and 
Table 1. Clinicopathological characteristics of the study patients

\begin{tabular}{|c|c|}
\hline Variable & Value \\
\hline Age (yr) & $59.0 \pm 10.9$ \\
\hline \multicolumn{2}{|l|}{ Sex } \\
\hline Male & $888(64.2)$ \\
\hline Female & $496(35.8)$ \\
\hline \multicolumn{2}{|l|}{ Pathologic stage } \\
\hline yp stage $0-2$ & $911(65.8)$ \\
\hline yp stage 3 & $473(34.2)$ \\
\hline \multicolumn{2}{|l|}{ Preoperative chemoradiotherapy } \\
\hline Yes & $536(38.7)$ \\
\hline No & $848(61.3)$ \\
\hline \multicolumn{2}{|l|}{ Surgical procedure } \\
\hline Abdominoperineal resection & $157(11.3)$ \\
\hline Hartmann procedure & $7(0.5)$ \\
\hline Sphincter preserving resection & $1,220(88.2)$ \\
\hline \multicolumn{2}{|l|}{ Adjuvant chemotherapy } \\
\hline Yes & $731(52.8)$ \\
\hline No & $653(47.1)$ \\
\hline \multicolumn{2}{|l|}{ Sarcopenia } \\
\hline Yes & $944(68.2)$ \\
\hline No & $440(31.8)$ \\
\hline \multicolumn{2}{|l|}{ Sarcopenic obesity } \\
\hline Yes & $307(22.2)$ \\
\hline No & $1,077(77.8)$ \\
\hline \multicolumn{2}{|l|}{ Visceral obesity } \\
\hline Yes & $670(48.4)$ \\
\hline No & $714(51.6)$ \\
\hline Body mass index $\left(\mathrm{kg} / \mathrm{m}^{2}\right)$ & $23.9 \pm 4.4$ \\
\hline$<18.5$ & $57(4.1)$ \\
\hline $18.5-23$ & $494(35.7)$ \\
\hline $23-25$ & $375(27.1)$ \\
\hline $25-30$ & $416(30.1)$ \\
\hline$>30$ & $42(3.0)$ \\
\hline Neutrophil-lymphocyte ratio & $2.33 \pm 1.68$ \\
\hline$<3$ & $1,099(79.8)$ \\
\hline$\geq 3$ & $278(20.2)$ \\
\hline
\end{tabular}

Values are presented as mean \pm standard deviation or number $(\%)$.

95\% confidence intervals. The covariates used in the analysis were sarcopenia, $\mathrm{SO}, \mathrm{VO}$, sex, age, pathologic tumor stage, obesity, PCRT, and inflammation. Variables with $\mathrm{p}<0.2$ were used in the multivariate analysis.

Subgroup analysis for evaluating the association between sarcopenia/SO and oncologic outcomes was performed according to the pathologic tumor stage (yp stage 0-2 and yp stage 3 ) and the inflammatory status (inflammation and no inflammation). Cancer stages were categorized using TNM tumor staging. We then analyzed whether sarcopenia and $\mathrm{SO}$ were associated with survival outcomes in these subgroups. The OS and RFS of each subgroup were analyzed by Kaplan-Meier curves and the HR was calculated using Cox regression analysis.

Statistical significance was reported at the $\mathrm{p}<0.05$ level. Statistical analyses were conducted using SPSS software ver. 21.0 (IBM Corp., Armonk, NY).

\section{Ethical statement}

The Institutional Review Board of the Asan Medical Center (2018-0993) approved this study. The informed consent was waived due to the retrospective design of this study.

\section{Results}

\section{Clinicopathological characteristics of the study patients}

Among the 1,384 rectal cancer patients included in the whole study cohort, $944(68.2 \%)$ were categorized as sarcopenia and $307(22.2 \%)$ as having SO. Majority of the patients were men $(\mathrm{n}=888,64.2 \%)$. The mean BMI was $23.9 \pm 4.4,458$ patients $(33.1 \%)$ were obese $\left(25 \mathrm{~kg} / \mathrm{m}^{2} \leq \mathrm{BMI}\right)$, and 42 patients $(3 \%)$ had a BMI of 30 or more. In addition, 670 patients $(48.4 \%)$ had VO and 536 subjects had undergone PCRT $(38.7 \%)$. In terms of the tumor pathology, 911 patients $(65.8 \%)$ had (y)p stage 0-2 tumor. Based on NLR, 278 patients (20.2\%) were categorized with inflammatory status. Table 1 summarizes these clinicopathological characteristics.

\section{Distribution and correlations among sarcopenia, BMI, and $\mathrm{VO}$}

Among the parameters, SMI, BMI, and visceral fat showed correlations. The SMI and BMI had a positive correlation $(\mathrm{r}=0.637, \mathrm{p}<0.001), \mathrm{BMI}$ and visceral fat had a slightly weaker positive correlation $(\mathrm{r}=0.444, \mathrm{p}<0.001)$, and SMI and visceral fat showed a weak positive correlation $(\mathrm{r}=0.247, \mathrm{p}<0.001)$ (Fig. 2). Incidence of sarcopenia and VO according to sex, age, and pathologic tumor stage was analyzed (Table 2). Sarcopenia and VO had a higher incidence in men. Patients older than 65 years were both more sarcopenic and had VO. The (y)p stage 0-2 cases also had a predominance of VO. The distribution of sarcopenia, obesity, or VO was not different according to receipt of PCRT. 

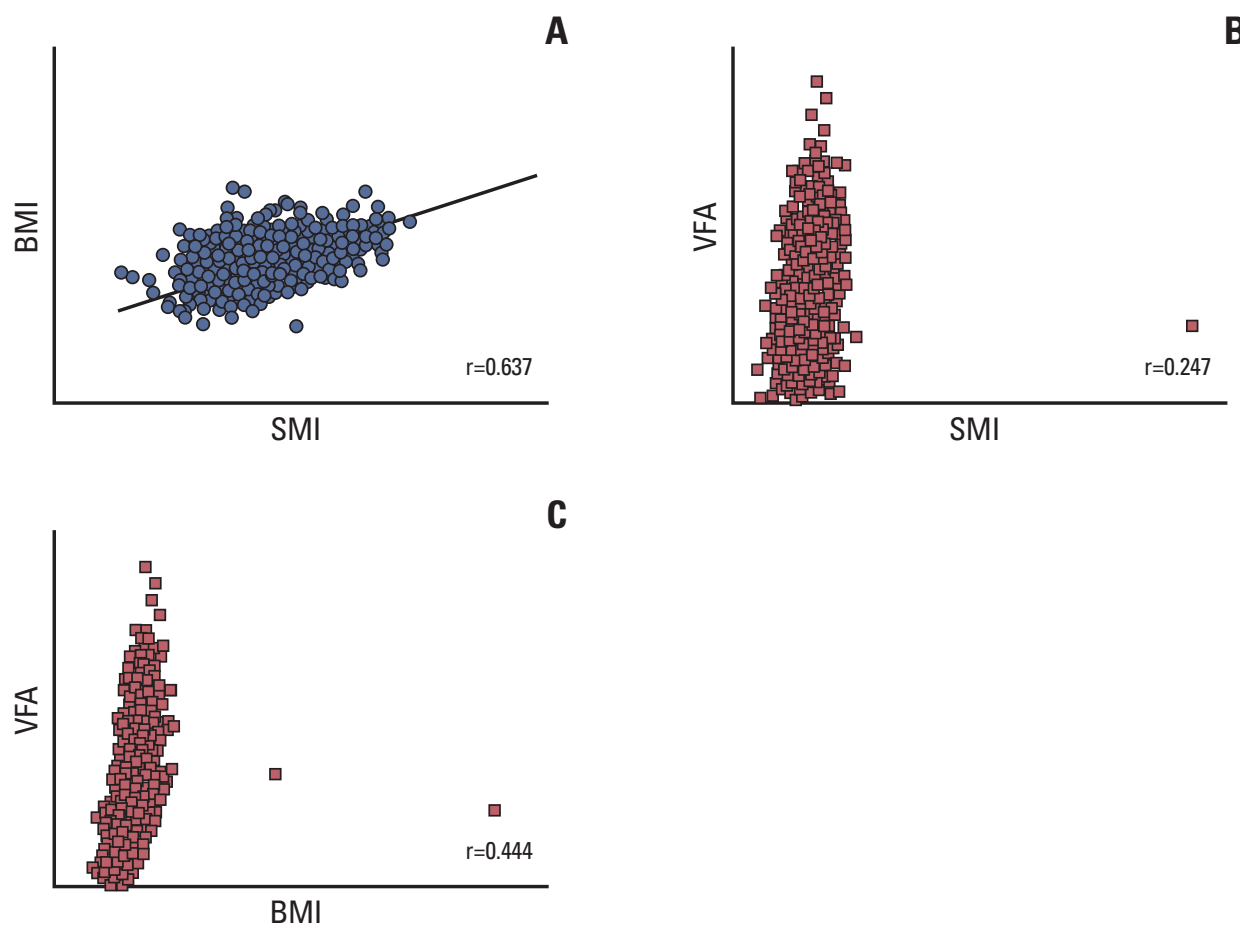

Fig. 2. (A-C) Correlation among skeletal muscle index (SMI) and body mass index (BMI), and visceral fat area (VFA). (A) The SMI and BMI showed a positive correlation $(\mathrm{r}=0.637)$.

Table 2. Distribution of sarcopenia, body mass index, and visceral fat according to sex, rectal tumor stage, and receipt of PCRT

\begin{tabular}{|c|c|c|c|c|c|c|c|c|c|}
\hline & \multicolumn{3}{|c|}{ Sarcopenia } & \multicolumn{3}{|c|}{ Obesity } & \multicolumn{3}{|c|}{ Visceral obesity } \\
\hline & No & Yes & p-value & No & Yes & p-value & No & Yes & p-value \\
\hline \multicolumn{10}{|l|}{ Sex } \\
\hline Male & $247(27.8)$ & $641(72.2)$ & $<0.001$ & $589(66.3)$ & $299(33.7)$ & 0.540 & $391(44.0)$ & $497(56.0)$ & $<0.001$ \\
\hline Female & $193(38.9)$ & $303(61.6)$ & & $337(67.9)$ & $159(32.1)$ & & $323(65.1)$ & $173(34.9)$ & \\
\hline \multicolumn{10}{|l|}{ Age (yr) } \\
\hline$<65$ & $362(38.6)$ & $575(61.4)$ & $<0.001$ & $621(66.3)$ & $316(33.7)$ & 0.502 & $518(55.3)$ & $419(44.7)$ & $<0.001$ \\
\hline$\geq 65$ & 78 (17.4) & $369(82.6)$ & & $305(68.2)$ & $142(31.8)$ & & $196(43.8)$ & $251(56.2)$ & \\
\hline \multicolumn{10}{|l|}{ yp stage } \\
\hline $0-2$ & $296(32.5)$ & $615(67.5)$ & 0.438 & $600(65.9)$ & $311(34.1)$ & 0.251 & $442(48.5)$ & $469(51.5)$ & 0.002 \\
\hline 3 & $144(30.4)$ & $329(69.6)$ & & $326(68.9)$ & $147(31.1)$ & & $272(57.5)$ & $201(42.5)$ & \\
\hline \multicolumn{10}{|l|}{ PCRT } \\
\hline No & $268(31.6)$ & $580(68.4)$ & 0.850 & $576(67.9)$ & $272(32.1)$ & 0.312 & $435(51.3)$ & $413(48.7)$ & 0.784 \\
\hline Yes & $172(32.1)$ & $364(67.9)$ & & $350(65.3)$ & $186(34.7)$ & & $279(52.1)$ & $257(47.9)$ & \\
\hline
\end{tabular}

Values are presented as number (\%). PCRT, preoperative chemoradiotherapy. 
A

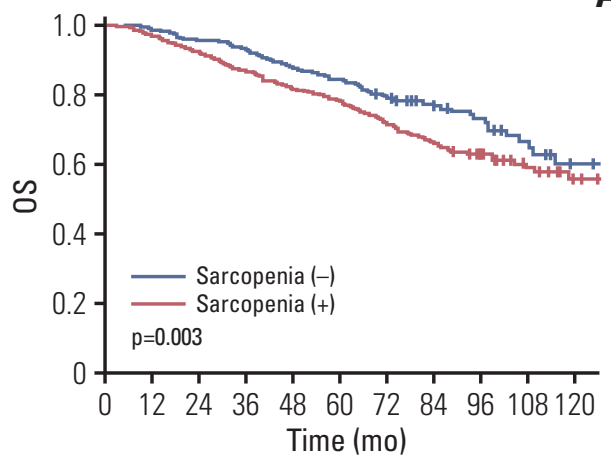

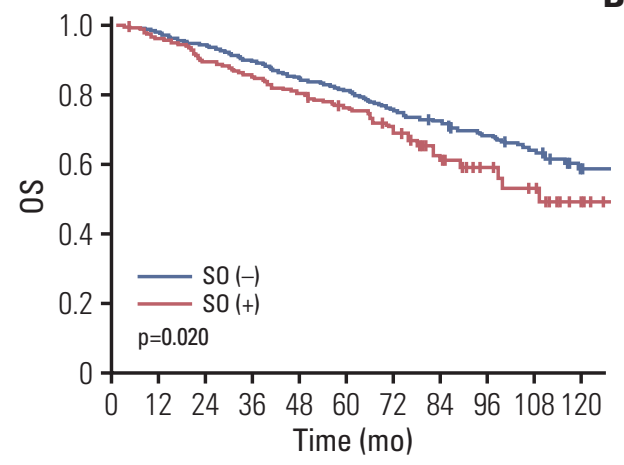

C

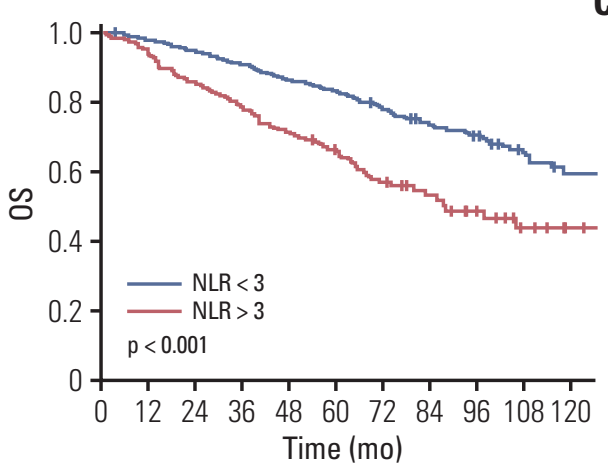

Fig. 3. Overall survival (OS) outcomes according to sarcopenia (A), sarcopenic obesity (SO) (B), and the neutrophil-lymphocyte ratio (NLR) (C). Sarcopenia and SO showed negative association with the 5-year overall survival rate. A high NLR was also associated with a poorer 5-year OS.

\section{Association of body composition and inflammatory sta- tus with oncologic outcomes}

The sarcopenic patients showed a significantly lower 5 -year OS rate ( $84 \%$ vs. $78 \%, \mathrm{p}=0.003)$, similar to the SO patients $(79.1 \%$ vs. $75.5 \% \mathrm{p}=0.020)$. Those with inflammation also displayed a significantly worse 5-year OS (83.3\% vs. $66.0 \%$, p < 0.001) (Fig. 3) and lower 5-year RFS (75.7\% vs. 55.4\%, p < 0.001). However, RFS showed no difference between patients categorized with sarcopenia and with SO. Moreover, the 5-year RFS and OS did not vary according to VO.

Sarcopenia, SO, visceral obesity, and obesity were not associated with RFS (Table 3). However, sarcopenia, SO, obesity, inflammatory status, age, sex, and pathologic stage were found to be associated with OS in univariate analysis. Obesity, SO, and inflammatory state were confirmed as independent factors associated with OS, together with age, sex, and pathologic stage, in multivariate analysis (Table 4).

\section{Influence of sarcopenia and SO on oncologic outcomes according to tumor stage and inflammatory status}

Oncologic outcomes according to sarcopenia and SO were analyzed in subgroups categorized by pathologic tumor stage and inflammatory status. For patients without lymph node metastasis ((y)p stage $0-2)$, the OS was significantly lower in the sarcopenia cases $(p=0.003)$, but no such differences were observed among the patients with lymph node metastasis (y)p stage 3$)(\mathrm{p}=0.310)$. SO patients with (y)p stage 0-2 also showed lower OS ( $\mathrm{p}=0.024)$, but this difference was not observed in SO patients with (y)p stage $3(\mathrm{p}=0.097)$. The $\mathrm{HR}$ of SO was higher in patients with (y)p stage $0-2$ (HR, 1.592) than in those with (y)p stage 3 (HR, 1.243) (Fig. 4).

In the no inflammatory status, an OS decline was observed in the sarcopenic cases $(86.3 \%$ vs. $81.7 \%$, p=0.014). For patients with inflammatory status, however, no OS differences were found. Whilst SO patients exhibited a lower OS with a no inflammatory status ( $84.5 \%$ vs. $79.4 \%, \mathrm{p}=0.006)$, there were no differences among the $\mathrm{SO}$ cases with an inflammatory state. In terms of OS, HR of SO was higher in no inflammatory status $(\mathrm{HR}, 1.576)$ than in inflammatory status $(\mathrm{HR}$, 
Table 3. Risk factors associated with recurrence-free survival

\begin{tabular}{|c|c|c|c|c|}
\hline \multirow{2}{*}{ Variable } & \multicolumn{2}{|c|}{ Univariate analysis } & \multicolumn{2}{|c|}{ Multivariate analysis } \\
\hline & Hazard ratio $(95 \% \mathrm{CI})$ & p-value & Hazard ratio $(95 \% \mathrm{CI})$ & p-value \\
\hline \multicolumn{5}{|c|}{ Visceral obesity } \\
\hline No & 1 & & - & \\
\hline Yes & $0.934(0.742-1.174)$ & 0.557 & - & - \\
\hline \multicolumn{5}{|c|}{ Sarcopenic obesity } \\
\hline No & 1 & & - & \\
\hline Yes & $1.026(0.778-1.351)$ & 0.858 & - & - \\
\hline \multicolumn{5}{|l|}{ Sarcopenia } \\
\hline No & 1 & & - & \\
\hline Yes & $1.001(0.784-1.278)$ & 0.992 & - & - \\
\hline \multicolumn{5}{|l|}{ Obesity } \\
\hline No & 1 & & - & \\
\hline Yes & $0.851(0.664-1.092)$ & 0.205 & - & - \\
\hline \multicolumn{5}{|l|}{ Age (yr) } \\
\hline$<65$ & 1 & & - & \\
\hline$\geq 65$ & $0.967(0.752-1.242)$ & 0.790 & - & - \\
\hline \multicolumn{5}{|c|}{ Pathologic stage } \\
\hline yp stage $0-2$ & 1 & & 1 & \\
\hline yp stage 3 & $3.334(2.640-4.210)$ & $<0.001$ & $3.547(2.803-4.488)$ & $<0.001$ \\
\hline \multicolumn{5}{|l|}{ Sex } \\
\hline Male & 1 & & - & \\
\hline Female & $0.932(0.732-1.186)$ & 0.566 & - & - \\
\hline \multicolumn{5}{|l|}{ PCRT } \\
\hline No & 1 & & 1 & \\
\hline Yes & $1.573(1.250-1.978)$ & $<0.001$ & $1.671(1.318-2.118)$ & $<0.001$ \\
\hline \multicolumn{5}{|c|}{ Inflammatory status } \\
\hline No & 1 & & 1 & \\
\hline Yes & $1.961(1.527-2.518)$ & $<0.001$ & $1.753(1.357-2.266)$ & $<0.001$ \\
\hline
\end{tabular}

CI, confidence interval; PCRT, preoperative chemoradiotherapy.

1.054) (Fig. 5).

\section{Discussion}

We found from our current analyses that $\mathrm{SO}$, low body mass index, and an inflammatory status are independent negative prognostic indicators for OS. Furthermore, our subgroup analysis demonstrated that association between OS and $\mathrm{SO}$ was more prominent in patients without lymph node metastasis or with no inflammatory status.

We analyzed the long-term outcomes of $\mathrm{SO}$ in our current rectal cancer patient series and were able to observe the effects of current practical treatment settings on survival outcomes by including the patients who had undergone PCRT, which has recently become a standard therapy for rectal cancer, and by considering combined treatment effects. Furthermore, by examining the associations between various body composition values including sarcopenia, $\mathrm{SO}, \mathrm{BMI}$, and $\mathrm{VO}$, and analyzing the effects of each of these parameters, we could evaluate those that had the most prognostic utility.

Studies on the association between body composition and long-term outcomes in colorectal cancer have shown inconsistent results. Sarcopenia has been reported as a risk factor for worse OS in some reports $[1,10,15]$, but, its influence on RFS was reportedly varied $[1,15]$. Some studies evaluating the influence of both body fat and skeletal muscle mass on oncologic outcomes indicated that $\mathrm{SO}$ is an independent predictor of poorer outcomes in colorectal cancer [4,14-16]. A recent study analyzed sarcopenia and systemic inflammation together and reported that these are independent factors for a decreased OS and RFS and are predictors of a higher risk if 
Table 4. Risk factors associated with overall survival

\begin{tabular}{|c|c|c|c|c|}
\hline \multirow{2}{*}{ Variable } & \multicolumn{2}{|c|}{ Univariate analysis } & \multicolumn{2}{|c|}{ Multivariate analysis } \\
\hline & Hazard ratio $(95 \% \mathrm{CI})$ & p-value & Hazard ratio $(95 \% \mathrm{CI})$ & p-value \\
\hline \multicolumn{5}{|c|}{ Visceral obesity } \\
\hline No & 1 & & - & \\
\hline Yes & $0.942(0.761-1.166)$ & 0.582 & - & - \\
\hline \multicolumn{5}{|c|}{ Sarcopenic obesity } \\
\hline No & 1 & & 1 & \\
\hline Yes & $1.332(1.043-1.702)$ & 0.022 & $1.395(1.067-1.822)$ & 0.015 \\
\hline \multicolumn{5}{|l|}{ Sarcopenia } \\
\hline No & 1 & & 1 & \\
\hline Yes & 1.415 (1.115-1.796) & 0.004 & $0.947(0.728-1.233)$ & 0.688 \\
\hline \multicolumn{5}{|l|}{ Obesity } \\
\hline No & 1 & & 1 & \\
\hline Yes & $0.682(0.536-0.868)$ & 0.002 & $0.639(0.494-0.828)$ & 0.001 \\
\hline \multicolumn{5}{|l|}{ Age (yr) } \\
\hline$<65$ & 1 & & 1 & \\
\hline$\geq 65$ & $2.113(1.702-2.622)$ & $<0.001$ & $2.205(1.762-2.760)$ & $<0.001$ \\
\hline \multicolumn{5}{|c|}{ Pathologic stage } \\
\hline yp stage $0-2$ & 1 & & 1 & \\
\hline yp stage 3 & $2.664(2.510-3.301)$ & $<0.001$ & $3.066(2.468-3.809)$ & $<0.001$ \\
\hline \multicolumn{5}{|l|}{ Sex } \\
\hline Male & 1 & & 1 & \\
\hline Female & $0.788(0.625-0.993)$ & 0.043 & 0.787 (0.624-0.992) & 0.043 \\
\hline \multicolumn{5}{|l|}{ PCRT } \\
\hline No & 1 & & - & \\
\hline Yes & $1.091(0.879-1.355)$ & 0.430 & - & - \\
\hline \multicolumn{5}{|c|}{ Inflammatory status } \\
\hline No & 1 & & 1 & \\
\hline Yes & $2.222(1.769-2.790)$ & $<0.001$ & $2.187(1.739-2.753)$ & $<0.001$ \\
\hline
\end{tabular}

CI, confidence interval; PCRT, preoperative chemoradiotherapy.

found in combination [10]. This study further discussed the effects of sarcopenia in concert with systemic inflammation on survival.

In the present study, the independent prognostic factors affecting OS were found to be $\mathrm{SO}$ and inflammatory status. There are studies reporting on the association between $\mathrm{SO}$ and poor OS and higher mortality associated with solid tumors $[17,18]$. The exact mechanism of how body composition (such as sarcopenia and SO) affects the survival of cancer patients remains unknown. Systemic inflammation might be a possible explanation [10]. A systemic inflammatory condition is known to increase the risk of cancer [19] and reduce a patient's response to treatment [20]. Sarcopenia and systemic inflammation are known to be correlated and this relationship would be substantial in obese patients who were defined as SO [5]. Possible explanations of how SO is independently related to mortality and morbidity might be the asso- ciations between immunity, inflammation, and myokines and adipocytokines $[5,21,22]$. Myokines play a pivotal role in cancer prevention as mediators of the beneficial effects of physical activity, counteracting the harmful effects of proinflammatory adipokines $[23,24]$. SO might be the condition bringing about the worsening of the imbalance in cytokines.

Our present subgroup analysis indicated an OS difference in SO patients and this was more pronounced in patients without lymph node metastasis or with no inflammatory status. This supports the hypothesis that systemic inflammation is a mechanism in which $\mathrm{SO}$ affects cancer survival. It may be possible that a severe inflammatory state itself has the principal negative effect on survival and that $\mathrm{SO}$ worsens this risk but relatively weakly. Although a non-inflammatory state accompanies SO, it might still progress into the aforementioned vicious cycle, exacerbate systemic inflammation, and thus have a relatively larger negative impact on survival. 


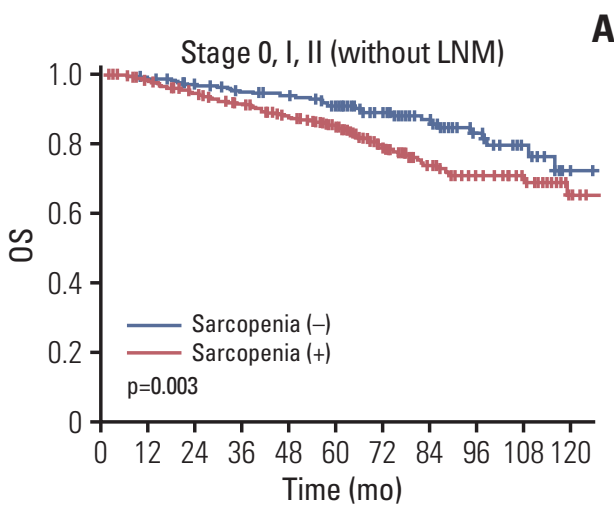

A
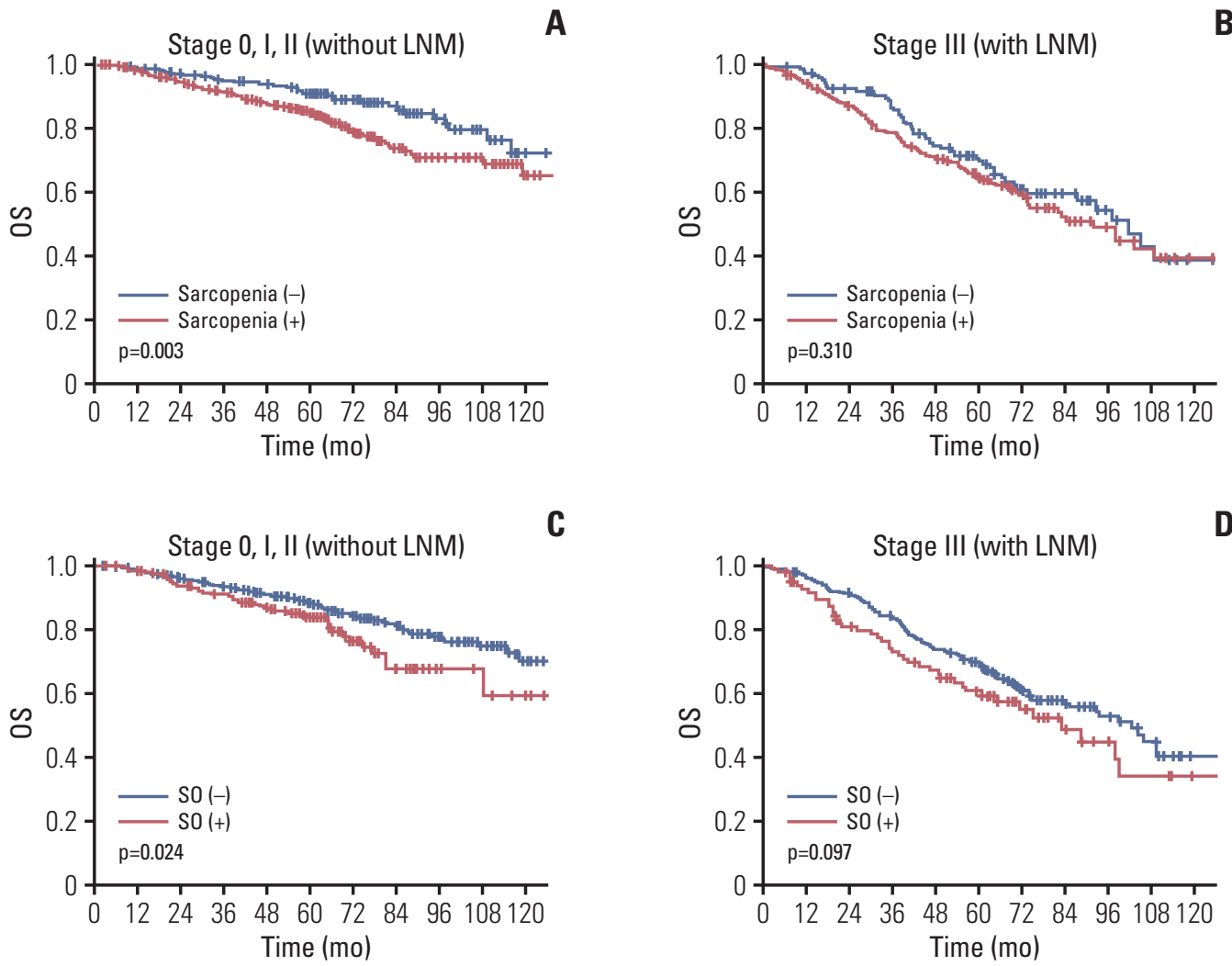

Fig. 4. Association between overall survival (OS) and sarcopenia and sarcopenic obesity (SO) according to the pathologic stage of the rectal tumor. The influence of sarcopenia on survival was evaluated in patients with (y)p stage $0-2(A)$ and $(y) p$ stage 3 (B) tumor. The association between SO and OS was also evaluated in the (y)p stage 0-2 (C) and (y)p stage 3 (D) rectal cancer cases. LNM, lymph node metastasis.

Since the advanced stage of CRC is also associated with a high inflammatory state $[25,26]$, the effects of stage without lymph node metastasis can possibly be explained in a manner similar to the no inflammatory state. Further studies will be needed to elucidate these possibilities.

In the present study, $\mathrm{SO}$ was associated with OS but not RFS. Obese patients are more likely to be affected by hypertension, diabetes, cardiac disease, and metabolic syndrome, and are usually associated with increased morbidity and mortality. Therefore, it was suspected that the adverse effect of $\mathrm{SO}$ on OS might be associated with medical co-morbidities rather than an effect of SO. In this study, however, we used $\mathrm{CT}$ measured parameters for the definition of $\mathrm{SO}$, not the combination of obesity and sarcopenia. Indeed, obesity itself was rather associated with better OS in the present study. Therefore, the negative effect of $\mathrm{SO}$ on OS was not considered to be mainly due to medical co-morbidities. Inflammatory status was the most potent associated factor of both RFS and OS in our cohort. Impact of SO on OS differed in degree according to the inflammatory status. Therefore, SO might be associated with OS through inflammation-related processes. Mechanism of how SO is associated with OS in colorectal cancer needs to be studied further.

Our present study had some limitations of note. First, since the definition of sarcopenia and SO is not fully established in Korea, we adopted the widely used Western definition. The prevalence of sarcopenia in CRC patients has been reported previously to range from $40 \%-60 \%[10,27,28]$, which is comparable to our present findings, but the cut-offs may not be appropriate for a Korean population since people in the West and East have a different baseline muscle mass or fat mass. Thus, our findings may have differed from those of other studies if a different baseline is adopted. Second, as we analyzed the effects of body mass composition parameters and inflammation based on single measurements prior to treatment, we were unable to examine postoperative changes over time. Furthermore, since our analyses were retrospective in nature and we had to use medical records to determine the inflammatory state, analysis of the effects of inflammatory markers is limited. 


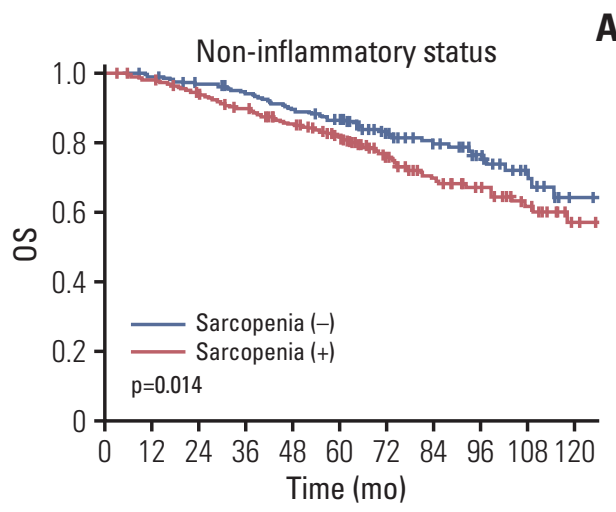

A
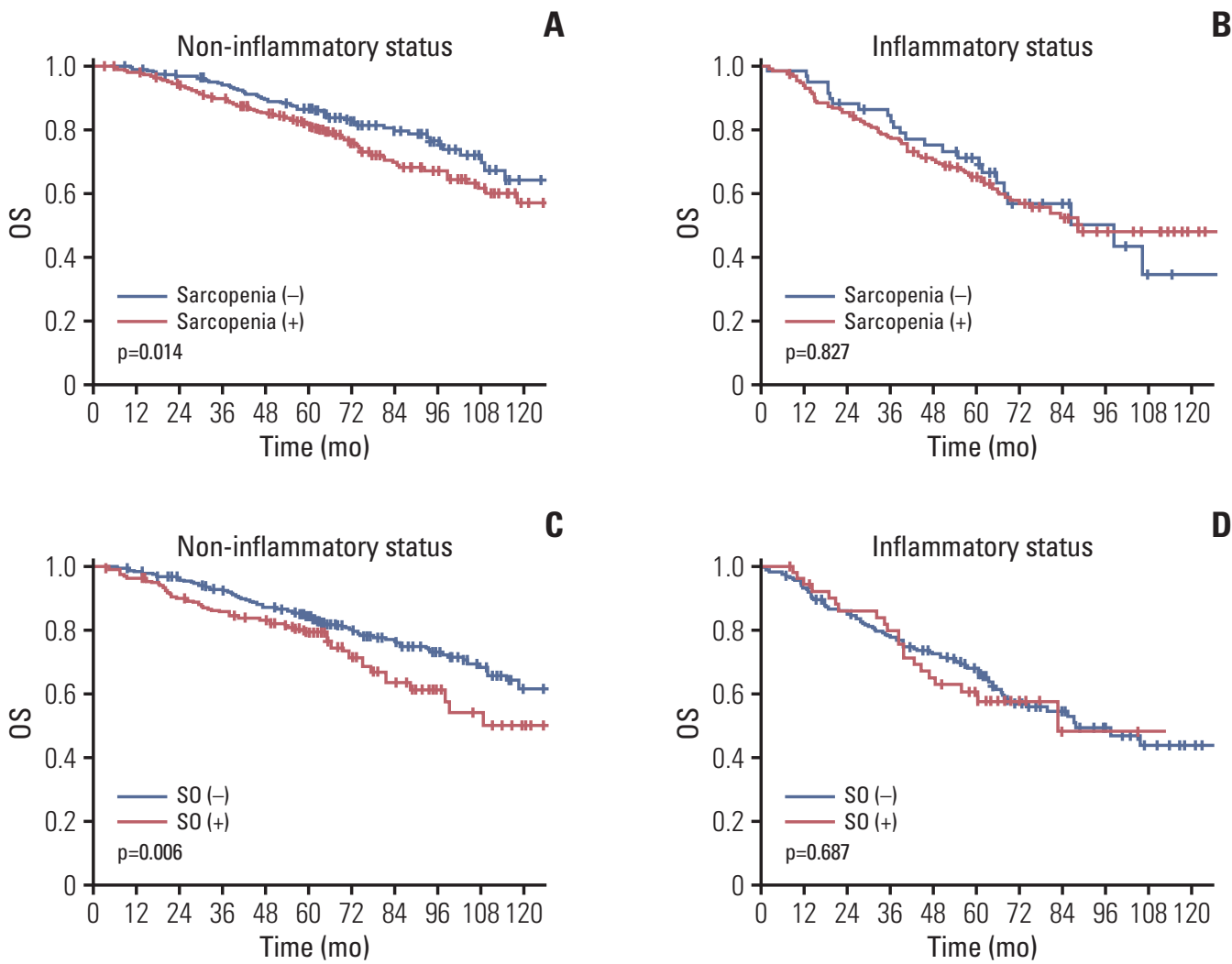

Fig. 5. Association between overall survival (OS) and sarcopenia and sarcopenic obesity (SO) according to the inflammatory status of the rectal cancer patients. The influence of sarcopenia on the OS rate in patients with a non-inflammatory (A) and inflammatory (B) status was assessed. The association between $\mathrm{SO}$ and OS outcomes in non-inflammatory (C) and inflammatory (D) status cases was also evaluated.

Notwithstanding these limitations, we enhanced the reliability of our data through the use of a larger-scale cohort that ensured greater statistical power. We thus believe that we have provided valid early study findings on the possibility of using $\mathrm{SO}$ as a prognostic factor in rectal cancer patients.

In conclusion, $\mathrm{SO}$ is a negative prognostic indicator in rectal cancer patients and is a more potent risk factor for poorer survival in patients with cancers without lymph node metastasis or those in a non-inflammatory state. Given that $\mathrm{SO}$ can be corrected, further prospective studies are warranted to determine if such interventions could improve cancer survival outcomes.

\section{Conflicts of Interest}

Conflict of interest relevant to this article was not reported.

\section{Acknowledgments}

This research was supported by a grant of the Korea Health Technology R\&D Project through the Korea Health Industry Development Institute (KHIDI), funded by the Ministry of Health \& Welfare, Republic of Korea (grant number: HI18C1216). 


\section{References}

1. Miyamoto Y, Baba Y, Sakamoto Y, Ohuchi M, Tokunaga R, Kurashige J, et al. Sarcopenia is a negative prognostic factor after curative resection of colorectal cancer. Ann Surg Oncol. 2015;22:2663-8.

2. Shachar SS, Williams GR, Muss HB, Nishijima TF. Prognostic value of sarcopenia in adults with solid tumours: a metaanalysis and systematic review. Eur J Cancer. 2016;57:58-67.

3. Haram A, Boland MR, Kelly ME, Bolger JC, Waldron RM, Kerin MJ. The prognostic value of neutrophil-to-lymphocyte ratio in colorectal cancer: a systematic review. J Surg Oncol. 2017;115:470-9.

4. Prado CM, Lieffers JR, McCargar LJ, Reiman T, Sawyer MB, Martin L, et al. Prevalence and clinical implications of sarcopenic obesity in patients with solid tumours of the respiratory and gastrointestinal tracts: a population-based study. Lancet Oncol. 2008;9:629-35.

5. Malietzis G, Johns N, Al-Hassi HO, Knight SC, Kennedy RH, Fearon $\mathrm{KC}$, et al. Low muscularity and myosteatosis is related to the host systemic inflammatory response in patients undergoing surgery for colorectal cancer. Ann Surg. 2016;263:320-5.

6. Black D, Mackay C, Ramsay G, Hamoodi Z, Nanthakumaran S, Park KG, et al. Prognostic value of computed tomography: measured parameters of body composition in primary operable gastrointestinal cancers. Ann Surg Oncol. 2017;24: 2241-51.

7. Hopkins JJ, Reif RL, Bigam DL, Baracos VE, Eurich DT, Sawyer MB. The impact of muscle and adipose tissue on long-term survival in patients with stage I to III colorectal cancer. Dis Colon Rectum. 2019;62:549-60.

8. Lieffers JR, Bathe OF, Fassbender K, Winget M, Baracos VE. Sarcopenia is associated with postoperative infection and delayed recovery from colorectal cancer resection surgery. Br J Cancer. 2012;107:931-6.

9. Huang DD, Wang SL, Zhuang CL, Zheng BS, Lu JX, Chen FF, et al. Sarcopenia, as defined by low muscle mass, strength and physical performance, predicts complications after surgery for colorectal cancer. Colorectal Dis. 2015;17:O256-64.

10. Feliciano EM, Kroenke CH, Meyerhardt JA, Prado CM, Bradshaw PT, Kwan ML, et al. Association of systemic inflammation and sarcopenia with survival in nonmetastatic colorectal cancer: results from the C SCANS study. JAMA Oncol. 2017;3: e172319.

11. Paik KY, Lee IK, Lee YS, Sung NY, Kwon TS. Clinical implications of systemic inflammatory response markers as independent prognostic factors in colorectal cancer patients. Cancer Res Treat. 2014;46:65-73.

12. Park JH, Watt DG, Roxburgh CS, Horgan PG, McMillan DC. Colorectal cancer, systemic inflammation, and outcome: staging the tumor and staging the host. Ann Surg. 2016;263:32636.

13. Bano G, Trevisan C, Carraro S, Solmi M, Luchini C, Stubbs B, et al. Inflammation and sarcopenia: a systematic review and meta-analysis. Maturitas. 2017;96:10-5.
14. Harimoto N, Shirabe K, Yamashita YI, Ikegami T, Yoshizumi $\mathrm{T}$, Soejima $\mathrm{Y}$, et al. Sarcopenia as a predictor of prognosis in patients following hepatectomy for hepatocellular carcinoma. Br J Surg. 2013;100:1523-30.

15. Choi MH, Oh SN, Lee IK, Oh ST, Won DD. Sarcopenia is negatively associated with long-term outcomes in locally advanced rectal cancer. J Cachexia Sarcopenia Muscle. 2018;9:53-9.

16. Baracos VE, Arribas L. Sarcopenic obesity: hidden muscle wasting and its impact for survival and complications of cancer therapy. Ann Oncol. 2018;29:ii1-9.

17. Carneiro IP, Mazurak VC, Prado CM. Clinical implications of sarcopenic obesity in cancer. Curr Oncol Rep. 2016;18:62.

18. Gruber ES, Jomrich G, Tamandl D, Gnant M, Schindl M, Sahora K. Sarcopenia and sarcopenic obesity are independent adverse prognostic factors in resectable pancreatic ductal adenocarcinoma. PLoS One. 2019;14:e0215915.

19. Coussens LM, Werb Z. Inflammation and cancer. Nature. 2002;420:860-7.

20. Chua W, Charles KA, Baracos VE, Clarke SJ. Neutrophil/lymphocyte ratio predicts chemotherapy outcomes in patients with advanced colorectal cancer. Br J Cancer. 2011;104:128895.

21. Tilg H, Moschen AR. Adipocytokines: mediators linking adipose tissue, inflammation and immunity. Nat Rev Immunol. 2006;6:772-83.

22. Xing SQ, Zhang CG, Yuan JF, Yang HM, Zhao SD, Zhang H. Adiponectin induces apoptosis in hepatocellular carcinoma through differential modulation of thioredoxin proteins. Biochem Pharmacol. 2015;93:221-31.

23. Trayhurn P, Drevon CA, Eckel J. Secreted proteins from adipose tissue and skeletal muscle: adipokines, myokines and adipose/muscle cross-talk. Arch Physiol Biochem. 2011;117: 47-56.

24. Pedersen BK, Febbraio MA. Muscles, exercise and obesity: skeletal muscle as a secretory organ. Nat Rev Endocrinol. 2012;8:457-65.

25. Leitch EF, Chakrabarti M, Crozier JE, McKee RF, Anderson $\mathrm{JH}$, Horgan PG, et al. Comparison of the prognostic value of selected markers of the systemic inflammatory response in patients with colorectal cancer. Br J Cancer. 2007;97:1266-70.

26. Liu Y, He X, Pan J, Chen S, Wang L. Prognostic role of Glasgow prognostic score in patients with colorectal cancer: evidence from population studies. Sci Rep. 2017;7:6144.

27. Broughman JR, Williams GR, Deal AM, Yu H, Nyrop KA, Alston SM, et al. Prevalence of sarcopenia in older patients with colorectal cancer. J Geriatr Oncol. 2015;6:442-5.

28. Reisinger KW, van Vugt JL, Tegels JJ, Snijders C, Hulsewe KW, Hoofwijk AG, et al. Functional compromise reflected by sarcopenia, frailty, and nutritional depletion predicts adverse postoperative outcome after colorectal cancer surgery. Ann Surg. 2015;261:345-52. 